\title{
Estudio de la expresión de cerbB-2 en el adenocarcinoma de prostata
}

\author{
P. San Miguel Fraile, J.E. Dos Santos*, E. Pélaez Boismorand, J.A. Ortiz Rey, \\ B. Iglesias Rodríguez, J. Cambronero Santos*, J.L. Fajardo Seijo, J.M. Rodríguez Costas, \\ M. Fernández Regueiro
}

Servicio de Anatomía Patológica. *Servicio de Urología. Centro Médico POVISA. Vigo. Pontevedra.

Actas Urol Esp 2005; 29 (1): 64-69

\section{RESUMEN}

ESTUDIO DE LA EXPRESIÓN DE cerbB-2 EN EL ADENOCARCINOMA DE PROSTATA

Objetivos: Determinar la expresión de la oncoproteína cerbB-2 en carcinomas de próstata con técnicas de inmunohistoquímica de próstata y comparar estos resultados con diversos factores pronósticos clínicos e histológicos.

Material y métodos: Se realizó tinción inmunohistoquímica con el anticuerpo cerbB-2 (DAKO) en 32 piezas de prostatectomia radical infiltradas por un adenocarcinoma. La expresión de cerbB-2 fue evaluada de 0 (no-tinción) a 3+ (tinción intensa y continua en la membrana celular) según protocolo. Se analizó la asociación estadística entre la expresión de cerbB-2 con algunos parámetros clínicos e histológicos.

Resultados: Se demostró positividad de membrana en 14 de las 32 neoplasias evaluadas (44\%). La sobreexpresión de cerbB-2 se correlacionó estadísticamente con el índice de Gleason $(\mathrm{p}=0.04)$ y estadio clínico $(\mathrm{p}=0.038)$.

Conclusiones: 1) Nuestro estudio muestra que aproximadamente el $44 \%$ de los carcinomas prostáticos expresan cerbB-2 con técnicas de inmunohistoquímica. 2) Esta serie permite deducir la necesidad de estandarizar la técnica inmunohistoquímica de cerbB-2 en el adenocarcinoma prostático. 3) En nuestro trabajo existe asociación estadística significativa de la expresión de cerbB2, según el método modificado, con el estadío clínico e índice de Gleason.

Palabras claves: Carcinoma de próstata. cerbB-2. Pronóstico. Prostatectomía radical.

\section{ABSTRACT}

EXPRESSION OF THE cerbB-2 (HER-2/neu) ONCOPROTEIN IN PROSTATIC ADENOCARCINOMA

Objectives: Our aim is to determine the expression of the cerbB-2 oncoprotein in prostate cancers using an immunohistochemistry staining and to compare these results with several clinical and histological prognostic factors.

Methods: An immunohistochemical staining using the cerbB-2 monoclonal antibody (Dako) was performed in 32 radical prostatectomy specimens diagnosed of adenocarcinoma. The intensity of cerbB-2 expression was evaluated with a scale that variated from 0 (no staining) to 3+ (strong complete membrane staining) according to published guidelines. Association of cerbB-2 index immunoreactivity with clinical and histological prognostic factors was examined.

Results: Definite positive membranous staining was detected in 14 of 32 neoplastic cases (44\%). Such overexpression was correlated with higher Gleason grade $(\mathrm{p}=0.04)$ and higher stage of disease $(\mathrm{p}=0.038)$.

Conclusions: 1) This study shows that $44 \%$ of all prostate cancer express the cerbB-2 oncoprotein with immunohistochemical technique. 2) These findings suggest that is necessary to standardize the immunohistochemical staining procedure with cerbB-2 in prostate adenocarcinoma. 3) The level of cerbB-2 expression was correlated with Gleason grade and clinical stage.

Key words: Prostate cancer. cerbB-2. Prognosis. Radical prostatectomy.

$\mathrm{E}$ 1 carcinoma de próstata es el tumor más frecuente en el hombre ${ }^{1}$ y la segunda causa más frecuente de muerte por cancer en el varón ${ }^{2}$. El pronóstico ha mejorado con el empleo de diferentes modalidades terapéuticas como la cirugía y la radioterapia; pero todavía los parámetros clínicos e histológicos no son suficientes para prever el pronóstico en todos los casos, ya que pacientes con características clínicas e histológicas similares tienen una evolución clínica muy diferente ${ }^{3}$. Por este motivo, se están estudiando nuevos marcadores moleculares. El estudio de oncogenes, genes supresores y otros factores reguladores del crecimiento tumoral han sido extensamente estudiados en múltiples neopla$\operatorname{sias}^{4-8}$, pero están insuficientemente analizadas en los tumores prostáticos.

El oncogen Her-2/neu, situado en el brazo largo del cromosoma 17, codifica una proteína transmembrana (p185HER2) con actividad tirosin-kinasa que actúa como un factor de crecimiento. Su alteración se traduce en una sobreexpresión en la membrana celular, generalmente secundaria a la amplificación del gen. Ha sido 
detectada con expresión variable, en una gran variedad de tumores malignos, como marcador pronóstico adverso, especialmente adenocarcinomas como: mama $(20 \%)^{5-8}$, ovario $(33 \%)^{6}$ y estómago $(20 \%)^{9}$.

En el carcinoma de próstata la sobreexpresión es muy variable ${ }^{10-25}$ y la causa de mayor discrepancia parece ser el método empleado ${ }^{23}$. Nosotros analizamos la sobreexpresión del oncogen cerbB-2 con técnicas de inmunohistoquímica en 32 piezas de prostatectomía y evaluamos su relación con diferentes variables histopatológicas y clínicas: estadio clínico, volumen tumoral, índice de Gleason, niveles de PSA, infiltración perineural y afectación ganglionar.

\section{MATERIAL Y MÉTODO}

\section{Selección de pacientes}

Se han estudiado retrospectivamente piezas de prostatectomías correspondientes a 32 pacientes diagnosticados de adenocarcinoma prostático entre los años 1998 y 2003; y que fueron tratados mediante cirugía con intención curativa. Ninguno de los pacientes recibió tratamiento hormonal y/o radioterapia previa a la cirugía. En todos los casos se revisaron las biopsias para confirmación del diagnóstico histológico y se realizó determinación de la expresión del oncogen cerbB2 en la sección más representativa del tumor.

La serie estudiada se compuso de 32 varones con un rango de edad entre 48 y 77 años y una edad media de 60,78 años El seguimiento medio fue de 34 meses (65 a 5 meses).

\section{Estudio histológico e inmunohistoquímico}

En el estudio histopatológico se consideraron los siguientes parámetros para su análisis: Volumen tumoral, grado de Gleason, estadio tumoral, infiltración perineural y afectación ganglionar.

$\mathrm{El}$ estadio tumoral, afectación ganglionar (metástasis o no), infiltración perineural (si o no) y volumen tumoral aproximado en la pieza de prostatectomía se categorizó tras el estudio histopatológico de las piezas quirúrgicas.

La determinación de la expresión del oncogen cerbB-2 se realizó en muestras de tejido tumoral incluidas en parafina. La técnica de inmunohistoquímica se realizó de dos formas:
- Método 1 (método estándar): Se utilizó la olla a presión durante 5 minutos en tampón citrato a pH6. Posteriormente se realizó una inhibición de la peroxidasa endógena con peróxido de hidrógeno al 3\% durante 10 minutos y se incubaron con el anticuerpo primario cerbB-2 (A0485, DAKO Diagnósticos S.A.) diluidos en proporción 1:200 con el anticuerpo diluyente (DAKO, 50809) durante 30 minutos. El método utilizado para la detección fue el de estreptoavidina-biotina marcada con peroxidasa y el cromógeno del revelado fue diaminobencidina (DAB).

- Método 2 (método modificado) ${ }^{23}$ : Se utilizó la olla a presión durante 5 minutos en tampón citrato a pH9. Posteriormente se realizó una inhibición de la peroxidasa endógena con peróxido de hidrógeno al 3\% durante 10 minutos y se incubaron con el anticuerpo primario cerbB-2 (A0485, DAKO Diagnósticos S.A.) diluidos en proporción 1:200 con el anticuerpo diluyente (DAKO, 50809) durante 30 minutos. El método utilizado para la detección fue el de estreptoavidina-biotina marcada con peroxidasa y el cromógeno del revelado fue DAB.

Valoración de la expresión inmunohistoquímica

Antes de proceder a la cuantificación de los resultados de inmunohistoquímica, se valoró en todos los casos la calidad de la técnica y se seleccionaron las áreas con más positividad, evitando mediciones en áreas periféricas, zonas desvitalizadas, con necrosis o artefacto. Todas las muestras fueron evaluadas sin saber en ningún momento de que pacientes procedían y la cuantificación de la técnica de inmunohistoquímica se realizó según está especificado en la Tabla 1.

\section{Tabla 1}

Esquema representativo de la valoración de la expresión de cerbB-2 con técnicas de inmunohistoquímica.

\begin{tabular}{ll}
\hline Resultados & Interpretación \\
\hline $\begin{array}{l}\text { Negativo: } \\
0\end{array}$ & - Ausencia de tinción \\
- $1+$ & $\begin{array}{l}\text { Tinción débil y discontinua en } \\
\text { membrana celular. }\end{array}$ \\
\hline Positivo: & Tinción moderada y continua en \\
- $2+$ & membrana celular \\
& $\begin{array}{l}\text { Tnción intensa y continua en membrana } \\
\text { celular. }\end{array}$ \\
\hline
\end{tabular}




\section{Estudio estadístico}

Para determinar la asociación entre las variables se empleó el test de $\chi^{2}$ o la prueba exacta de Fisher. Previamente las variables fueron estratificadas de la siguiente forma:

- Índice de Gleason: 5, 6, 7, 8, 9.

- Estadio Tumoral: pT2a, pT2b, pT2c, pT3a, pT3b, pT4.

- Infiltración perineural: si, no.

- Afectación ganglionar: si, no.

- Volumen tumoral: $\leq 15 \%, 15-30 \%,>30 \%$.

- Niveles de PSA: $\leq 10,10-15,>15$.

Como nivel de significación estadístico se consideró un valor de $\mathrm{p}(\leq 0,05$.

\section{RESULTADOS}

Las características clínico-patológicas de los 32 casos de adenocarcinoma prostáticos evaluados se muestran en la Tabla 2. Con el método

\section{Tabla 2}

Relación estadística de la expresión de cerbB-2 con diferentes parámetros clínicos e histológicos.

\begin{tabular}{|c|c|c|c|}
\hline & $\begin{array}{c}\text { cerB-2 } \\
\text { positivo } \\
14 \text { casos } \\
(45 \%)\end{array}$ & $\begin{array}{c}\text { cerB-2 } \\
\text { negativo } \\
17 \text { casos } \\
(55 \%)\end{array}$ & Valor $\mathbf{p}$ \\
\hline \multicolumn{4}{|l|}{ Gleason } \\
\hline 5 & 0 & 1 & \multirow{5}{*}{$P=0,04$} \\
\hline 6 & 1 & 3 & \\
\hline 7 & 7 & 13 & \\
\hline 8 & 3 & 0 & \\
\hline 9 & 3 & 0 & \\
\hline \multicolumn{4}{|l|}{ Estadio } \\
\hline pT2A & 0 & 3 & \multirow{6}{*}{$P=0,038$} \\
\hline pT2B & 0 & 5 & \\
\hline pT2C & 4 & 3 & \\
\hline pT3A & 5 & 5 & \\
\hline pT3B & 4 & 1 & \\
\hline pT4 & 1 & 0 & \\
\hline \multicolumn{4}{|c|}{ Infiltración perineural } \\
\hline $\mathrm{Si}$ & 14 & 14 & \multirow{2}{*}{$\mathbf{P}=\mathbf{0 , 1 0}$} \\
\hline No & 0 & 3 & \\
\hline \multicolumn{4}{|c|}{ Afectación ganglionar } \\
\hline Sí & 1 & 1 & \multirow{3}{*}{$P=0,75$} \\
\hline No & 8 & 15 & \\
\hline No conocido & 5 & 1 & \\
\hline \multicolumn{4}{|c|}{ Volumen tumoral } \\
\hline$\leq 15 \%$ & 5 & 4 & \multirow{3}{*}{$P=0,74$} \\
\hline $15-30 \%$ & 6 & 9 & \\
\hline$>30 \%$ & 3 & 4 & \\
\hline \multicolumn{4}{|c|}{ Niveles de PSA } \\
\hline$\leq 10$ & 10 & 8 & \multirow{4}{*}{$\mathbf{P}=\mathbf{0 , 2 8}$} \\
\hline $10-15$ & 0 & 3 & \\
\hline$>15$ & 1 & 3 & \\
\hline No conocido & 3 & 3 & \\
\hline
\end{tabular}

de inmunohistoquímica estándar no se demostró positividad en la expresión para cerbB-2 en ningún caso ( 3 casos $1+, 29$ casos 0 ) y se demostró positividad en la expresión para cerbB-2 según el método modificado de Sánchez et al ${ }^{22}$ en 14 casos (44\%), mientras que en 17 casos (53\%) la tinción fue negativa y en un caso fue no valorable (3\%) (Tabla 3). Las Figuras 1 y 2 representa ejemplos de negatividad (a y b) y positividad (c y d) para cerbB-2. Para el estudio estadístico únicamente se valoraron los resultados obtenidos según el método modificado.

No se demostró asociación estadística significativa entre la expresión de cerbB-2 en el tumor con la infiltración perineural, afectación ganglionar, volumen tumoral y niveles de PSA. Sin embargo, si observamos asociación estadística significativa entre la expresión de cerbB-2 con el indice de Gleason $(\mathrm{p}=0,04)$ y el estadio tumoral $(\mathrm{p}=0,038)$.

En nuestra serie se observó una relación directa entre el índice de Gleason y el estadio tumoral con la expresión de cerbB-2 en las células tumorales. De forma que cuanto mayor era el índice de Gleason, o el estadio del tumor era más avanzado aumentaba la expresión de cerbB-2. En el grupo de pacientes con estadio tumoral pT2 4 de los 15 pacientes (26\%) mostraban amplificación para cerbB-2 en sus células tumorales, mientras que en los casos con estadio pT3 9 de los 15 pacientes (60\%) mostraban amplificación para cerbB-2 y el 100\% para el estadio pT4 (Fig. 3). Algo similar ocurre con el indice de Gleason apreciándose una amplificación del 0\% para los tumores con índice de Gleason 5, una amplicación del 100\% para los tumores con índice de Gleason 8 y 9 y una ampli-

\section{Tabla 3}

Comparación de los resultados según los dos métodos de inmunohistoquímica analizados.

\begin{tabular}{lcc}
\hline $\begin{array}{l}\text { Resultados } \\
\text { modificado }\end{array}$ & $\begin{array}{c}\text { Método estándar } \\
\text { (casos) }\end{array}$ & $\begin{array}{c}\text { Método } \\
\text { (casos) }\end{array}$ \\
\hline Negativo: & 29 & \\
0 & 3 & 1 \\
$1+$ & 0 & 16 \\
\hline Positivo: & 0 & 7 \\
$2+$ & 0 & 7 \\
$3+$ & & 1 \\
\hline No valorables: & &
\end{tabular}



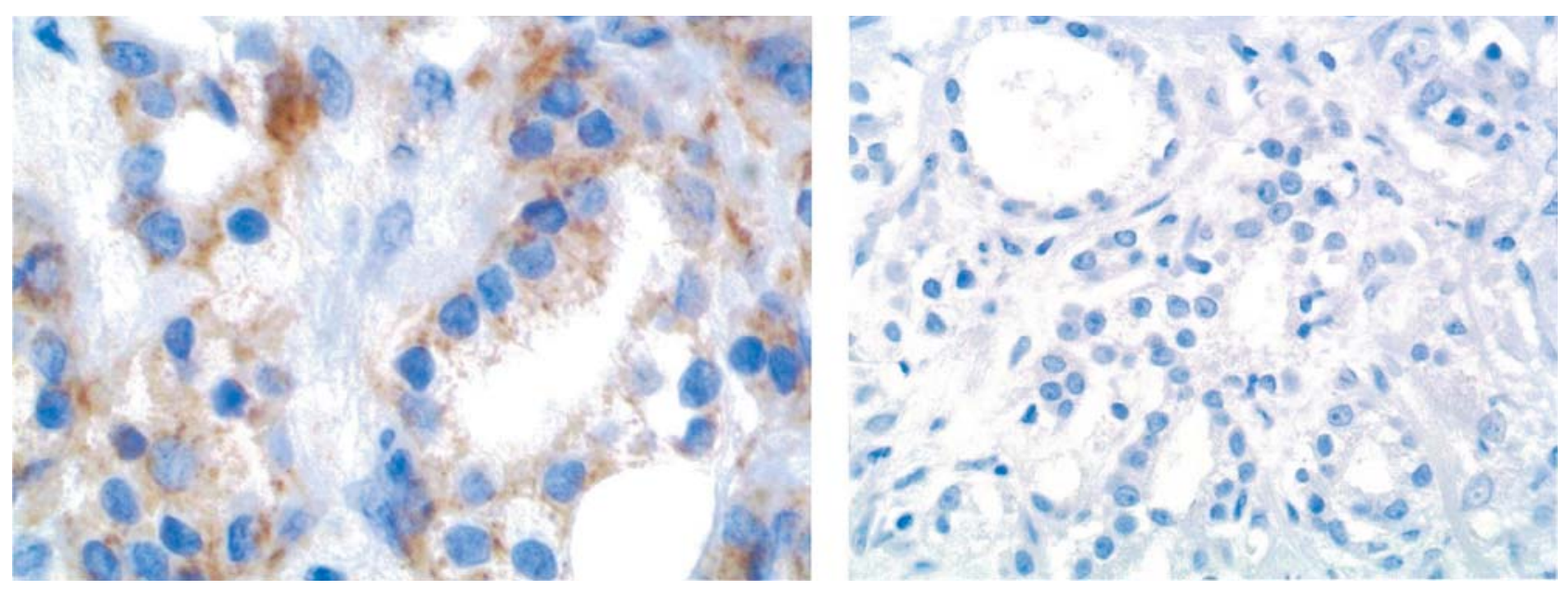

FIGURA 1. Ejemplo de tinción inmunohistoquimica para cerbB-2: Adenocarcinoma de próstata con expresión de membrana negativa ( 0 y $1+$ ).
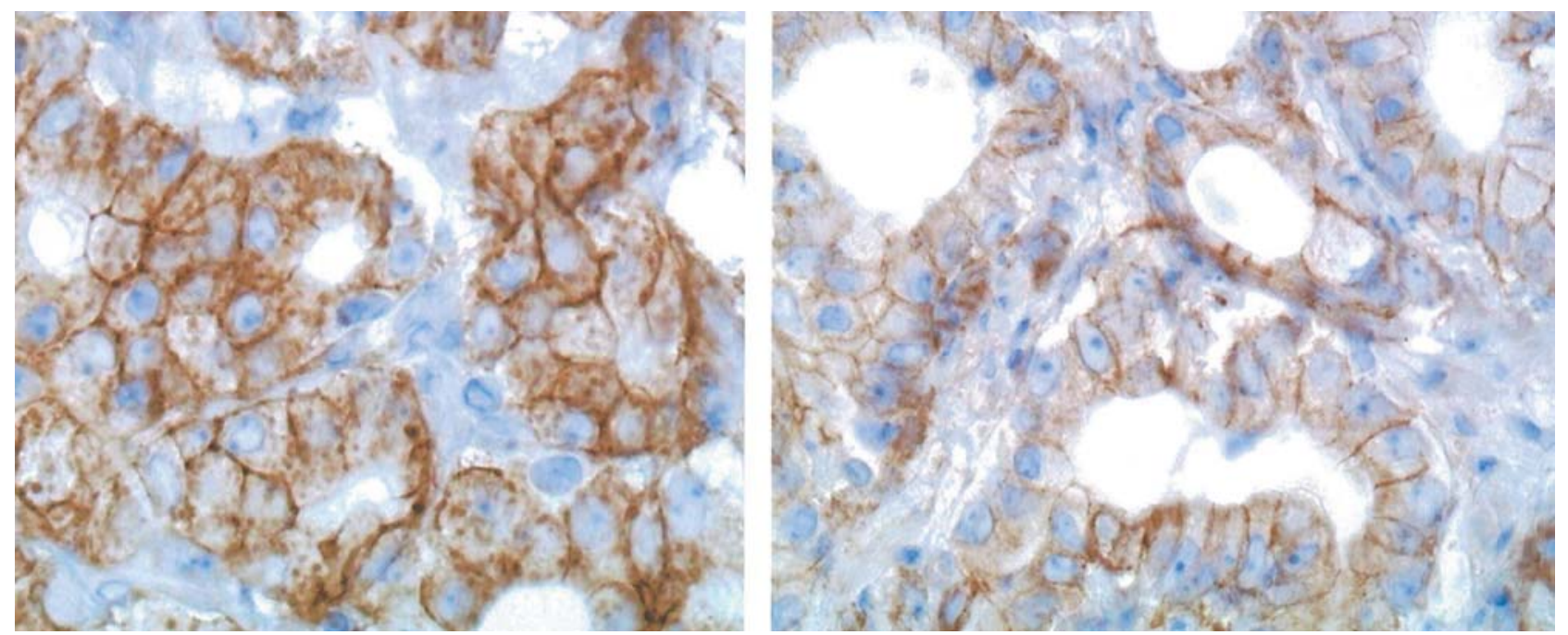

FIGURA 2. Ejemplo de inmunohistoquímica para cerbB-2: Adenocarcinoma de próstata con expresión de membrana positiva $(2+y+3)$.

ficación del $22 \%$ y $35 \%$ para los tumores con índice de Gleason 6 y 7 respectivamente (Fig. 4).

\section{DISCUSIÓN}

Este estudio tiene un doble objetivo: evaluar los métodos semicuantitativos de expresión del cerbB-2 con técnicas de inmunohistoquímica en pacientes con adenocarcinoma de próstata y analizar la correlación entre la expresión de cerbB-2 detectadas por técnicas de inmunohistoquímica con las variables pronósticas clásicas.

La expresión de cerbB-2 en adenocarcinomas prostáticos es muy variable (rango 0-100\%) ${ }^{10-25}$, con un porcentaje medio en la mayoría de los estudios que oscila entre el 25 y el $40 \%{ }^{24}$. Esta discrepancia en las series se debe principalmente a la gran heterogeneidad de las mismas y a la diversidad de métodos empleados para su determinación ${ }^{23}$.

Los resultados con los dos métodos inmunohistoquímicos analizados fueron muy diferentes. Con la técnica estándar, no se demostró positividad en ningún caso; mientras que con la técnica modificada, la expresión fue del $44 \%$. Estos resultados son totalmente superponibles a los aportados por Sánchez et $\mathrm{al}^{23}$, que según la literatura revisada, es el único trabajo en el que también se analiza la expresión de este marcador 


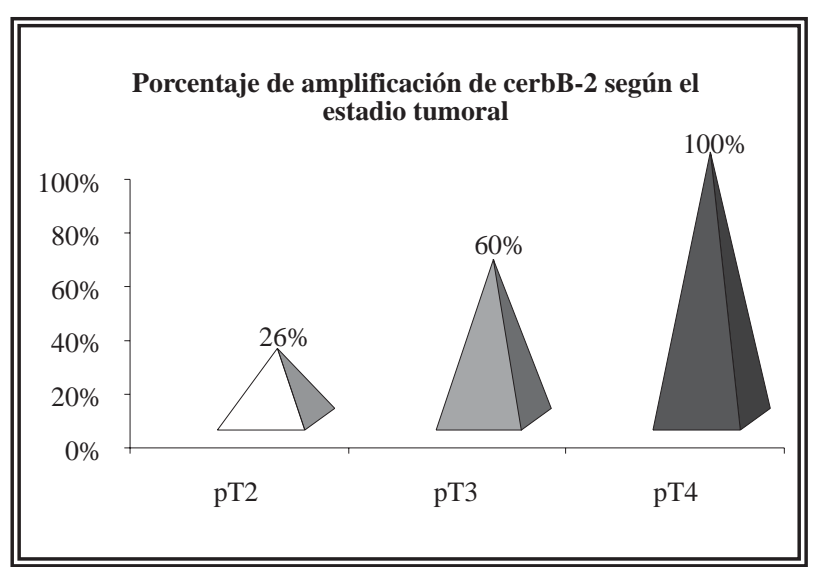

FIGURA 3. Porcentaje de amplificación de cerbB-2 según el estadio tumoral.

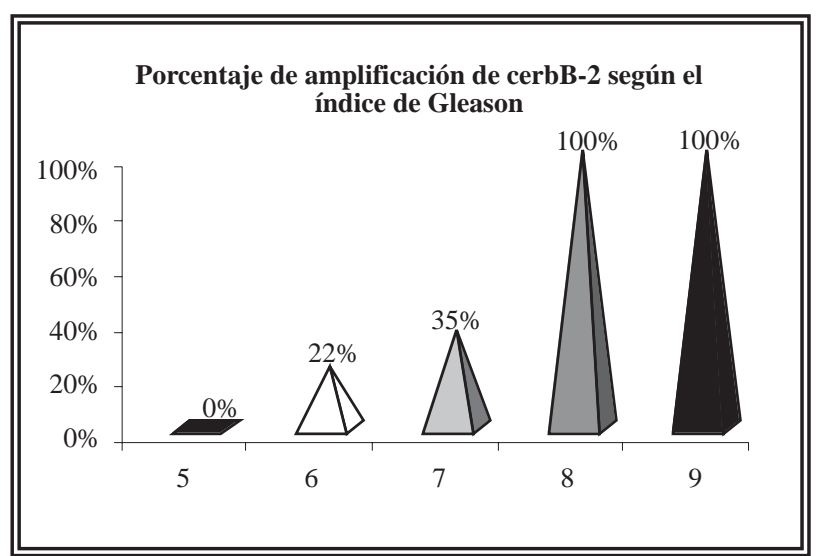

FIGURA 4. Porcentaje de amplificación de cerbB-2 según el grado de Gleason

con dos métodos inmunohistoquímicos diferentes. En este último, la expresión media de cerbB-2 fue del 3 y 50\% con el método estándar y modificado, respectivamente. Nuestra serie y la de Sánchez et $\mathrm{al}^{23}$ nos permite deducir la necesidad de estandarizar la técnica en función del tipo de tejido a analizar con la finalidad de disminuir el número de falsos negativos.

La expresión de cerbB-2 es un factor pronóstico de máxima importancia en varias neoplasias como el cáncer de cérvix ${ }^{9}$, mama $^{10-12}$, ovario ${ }^{11} \mathrm{y}$ estómago $^{13}$. El cáncer de próstata, no parece ser una excepción, aunque los resultados hayan sido menos concluyentes. En la próstata la expresión de cerbB-2 es mayor en los tumores metastásicos que en los localizados y su expresión podría ser un marcador de progresión tumoral ${ }^{22}$. Además son varias las publicaciones previas, con series amplias y estudios estadísticos que han investigado el valor pronóstico de la expresión de cerbB2 y su asociación con otros parámetros clínicopatológicos ${ }^{10,15,16,22,23}$. En algunos de estos estudios se confirma que existe asociación estadística entre la expresión de la oncoproteína y el estadio clínico $^{15,16,23}$. En este sentido, nuestra serie es equiparable $(\mathrm{p}=0.038)$ y el porcentaje de tumores con expresión para el cerbB-2 según el estadio clínico fue del 27\%, 60\% y 100\% para los tumores en estadio pT2, pT3 y pT4, respectivamente. Estos datos sugieren que la expresión de cerbB-2 es un factor pronóstico adverso en el adenocarcinoma prostático que este asociado a la progresión tumoral, ya que su expresión es mayor en los tumores con estadios clínicos más avanzados.

Con respecto la relación entre el índice de Gleason y la expresión de cerbB-2, nuestros resultados son similares a los aportados por Ware $^{10}$ y Sánchez ${ }^{23}$. Nosotros observamos mayor expresión de cerbB-2 en los tumores con mayor indice de Gleason. Otros autores 14,15,22, 24 no demostraron correlación entre la expresión de la oncoproteína y el índice de Gleason, aunque sí, con el pronóstico del tumor. Las discrepancias entre las series pueden reflejar heterogeneidad en la selección de los pacientes ${ }^{24}$, tratamientos ${ }^{24}$ y técnicas de inmunohistoquímica empleadas ${ }^{23}$.

Por último, en nuestra serie no encontramos asociación estadística de la expresión de la oncoproteína con la infiltración perineural, afectación ganglionar, volumen tumoral y niveles de PSA. Tan sólo dos autores ${ }^{22,26}$ han encontrado una relación directa entre que la expresión de cerbB2 y los niveles de PSA. Estos resultados deben de ser confirmados mediante estudios en series más amplias. En lo que conocemos, ningún estudio ha analizado la asociación entre expresión de cerbB-2 y la infiltración perineural, afectación ganglionar y volumen tumoral. Morote et al no observaron diferencias en la inmunotinción con el cerbB-2 y la existencia de metástasis óseas o extraóseas.

En resumen, nuestros resultados:

- Sugieren la necesidad de estandarizar la técnica inmunohistoquímica de cerbB-2 en función del tipo de tejido a analizar, con la finalidad de disminuir el número de falsos negativos. 
- La expresión de cerbB-2 podría estar relacionado con el estadio tumoral e índice de Gleason en el adenocarcinoma de próstata. Estos resultados deben ser confirmados mediante estudios en series más amplias.

\section{REFERENCIAS}

1. Boring CC, Squires TS, Tong T. Cancer Statistics 1991; $\mathrm{Ca}, 41: 19$.

2. Jemal A, Thomas A, Murray T, Thun M. Cancer Statistics, 2002. CA Cancer J Clin 2002;52:23-47.

3. Mydlo JH, Kral JG, Volpe M, Axotis C, Macchia RJ, Pertschuk LP. An analysis of microvessel density, androgen receptor, p53 and HER-2/neu expression and Gleason score in prostate cancer. Preliminary results and therapeutic implications. Eur Urol 1998;34:426-432.

4. Hale R, Buckley C, Fox H, Williams J. Prognostic value of c-erbB-2 expression in uterine cervical carcinoma. J Clin Pathol 1992;45:594-596.

5. Wright C, Angus B, Nicholson S et al. Expression of cerbB-2 oncoprotein: a prognostic indicator in human breast cancer. Cancer Res 1989;49:2087-2090.

6. Slamon DJ, Godolphin W, Jones LA, et al. Studies of HER$2 /$ neu proto-oncogene in human breast and ovarion cancer. Science 1989;244:707-712.

7. Borg A, Tandon AK, Sigurdsson H, et al. HER-2/ neu amplification predicts poor survival in node-positive breast cancer. Cancer Res 1990;50:4332-4337.

8. Koeppen HKW, Wright BD, Burt AD, et al. Overexpression of HER2/neu in solid tumours: an immunohistochemical survey. Histopathology 2001;38:96-104.

9. Falck VG, Gullick WJ. c-erbB-2 oncogene product staining in gastric adenocarcinoma. An immunohistochemical study. J Pathol 1989;159:107-111.

10. Ware JL, Maygarden SJ, Koontz WW, Strom S. Immunohistochemical detection of c-erbB-2 protein in human benign and neoplastic prostate. Hum Pathol 1991;22:254258.

11. Mellon K, Thomston S, Charlton RG, et al. P53, c-erbB-2 and the epidermal growth factor receptor in the benign and malignant prostate. J Urol 1992;147:496-499.

12. Berner A, Nesland JM, Waehre H, Side J, Fossa SD. Hormone resistant prostatic adenocarcinoma: an evaluation of prognostic factors in pre- and post-treatment specimens. Br J Cancer 1993;68:380-384

13. Kokuho M, Yoshiki R, Hamaguchi A, Okada Y, Tomoyhosi T, Higuchi K. Immunohistochemical study of c-erbB-2 protooncogene product in prostatic cancer. Jpn J Urol 1993; 84:1872-1878.

14. Giri DK, Wadhwa SN, Upadhaya SN, Tawar GP. Expression of NEU/HER-2 oncoprotein (p185neu) in prostate tumors: an immunohistochemical study. Prostate 1993;23:329-336.
15. Sadasivan R, Morgan R, Jennings S, et al. Over-expression of HER-2/NEU may be an indicator of poor prognosis in prostate cancer. J Urol 1993;150:126-131.

16.Kuhn EJ, Kurnot RA, Sesterhenn IA, Chang EH, Moul JW. Expression of the c-erbB-2 (Her-2/neu) oncoprotein in prostate cancer. J Urol 1993;150:1427-1433.

17. Myers RB, Srivastava S, Oelschlager DK, Grizzle WE. Expression of p160erbB-3 and p185erbB-2 in prostatic intraepithelial neoplasia and prostatic adenocarcinoma. J Natl Cancer Inst. 1994;86:1140-1145.

18. Fox SB, Persad RA, Coleman N, Day CA, Silcocks PB, Collins CC. Prognostic value of c-erbB-2 and epidermal growth factor receptor in stage Al (Tla) prostatic adenocarcinoma. Br J Urol 1994;74:214-220.

19. Lyne JC. Naraghi RM, Melhem MA et al. Human prostate cancer cells respond to but do not express neu differentiation factor (NDF) in spite of HER2/neu gene amplification and protein overexpression. Cancer J Sci Am 1995;3:21-30.

20. Cohen RJ, Cooper K, Haffejee Z, Robinson E, Becker PJ. Immunohistochemical detection of oncogene proteins and neuroendocrine differentiation in different stages of prostate cancer. Pathology 1995;27:229-232.

21. Gu K, Mes-Masson A, Gauthier J, Saad F. Overexpression of Her-2/neu in human prostate cancer and benign and malignant prostate. Cancer 1996;99:185-189.

22. Morote J, Torres I, Caceres C, Vallejo C, Schwartz S, Reventos J. Prognostic value of immunohistochemical expression of the c-erbB-2 oncoprotein in metastasic prostate cancer. Int J Cancer 1999;84:421-425.

23. Sánchez KM, Sweeney CJ, Mass R, et al. Evaluation of HER-2/neu expression in prostatic adenocarcinoma. A request for a standardized, organ specific methodology. Cancer 2002;95:1650-1655.

24. Fossa A, Lilleby W, Fossa SD, Gaudernack G, Torlakovic G, Berner A. Independent prognostic significance of HER2 oncoprotein expression in pNO prostate cancer undergoing curative radiotherapy. Int J Cancer 2002;99:100105.

25. Lara PN, Meyers FJ, Gray CR, et al. Her-2/neu is overexpressed infrequently in patients with prostate carcinoma. Results from california cancer consortium screening trial. Cancer 2002;94:2584-2589.

26. Zhau HE, Wan DS, Zhou J, Miller GJ, Von Eschenbach AC. Expression of cerbB-2/neu proto-oncogene in human prostatic-cancer tissues and cell lines. Mol Carcinogen 1992;5:320-327.

Dra. P. San Miguel Fraile

Servicio de Anatomía Patológica.

Centro Médico POVISA. C/ Salamanca, no 5 . 36211 Vigo (Pontevedra).

(Trabajo recibido el 1 junio de 2004) 